\title{
IMPROVEMENT IN PROPERTIES OF CHROMIUM COATED ABS PARTS
}

\author{
SUNIL GAEKWAD ${ }^{1 *} \&$ DR. SANTOSH KUMAR A. $\mathbf{N}^{2}$ \\ ${ }^{I}$ Department of Mechanical Engineering, the National Institute of Engineering, Mysuru, Karnataka, India \\ ${ }^{2}$ Departmen of Mechanical Engineering, Sri Jayachamarajendra College of Engineering, Mysuru, Karnataka, India
}

\begin{abstract}
Fused Deposition Modelling (FDM) creates parts with complex designs by extruding layers of polymers, such as acrylonitrile butadiene styrene (ABS) one on top of another. All parts printed using FDM have presented with a rough surface. There are numerous parameters that influence the thermo-mechanical properties of the FDM parts and further study is needed to fully comprehend their inter relationship. Most studies agree on the fact that coating will improve its mechanical properties. However, very few standards have been established. An attempt has been made to coat ABS parts with a chromium based paint in order to determine the significance of the coating. Tests conducted on the coated parts have shown an increase in hardness and impact strength while the surface roughness has reduced.

KEYWORDS: Rapid Tooling, Fused Deposition Modelling, Additive Manufacturing, Surface Roughness, Thermo Mechanical Properties \& Chromium Coating
\end{abstract}

Received: Jun 08, 2020; Accepted: Jun 28, 2020; Published: Sep 15, 2020; Paper Id.: IJMPERDJUN20201253

\section{INTRODUCTION}

Started during the early 1980s, Rapid prototyping (RP), also called Additive Manufacturing (AM), is a set of processes used to fabricate components directly with the aid of computer-aided design software(CAD). [1]

Additive Manufacturing (AM) is the fabrication of three dimensional physical models by the application of material in successive layers. The models created from AM can range from form-fit prototypes to fully functional test mules and products. The AM processes are used because of their quick turnover time compared to conventional techniques of manufacturing. Their applications range from everyday products like bookmarks to medical implants and aerospace components.

The growth of $\mathrm{AM}$ is at such a pace that the creative progress of 3D printing towards design \& manufacture of products will constantly produce a variety of new avenues with far reaching capabilities. [1]

These systems are categorised on the basis of build material being used, namely, Liquid, Solid \& Powder. The two most commonly used systems are Stereolithography, in which a photosensitive polymer stored in a large vat is cured using lasers to obtain the finished article and Fused Deposition Modelling, where thermoplastic filaments are extruded from a heated tip to create the product one layer at a time. [2]

\section{FUSED DEPOSITION MODELLING (FDM)}

In FDM, the raw material in the form of filaments is fed through rollers and a heating element so that it melts and the melt is extruded out of nozzles. The first layer adheres to the build platform and all successive layers adhere to the previous ones after which the layer cools and hardens. The motion of the extrusion head, which creates they layers of plastics, is restricted to the XY plane. The build platform will move down by a distance equal to the thickness of 
each layer so that the newer layers can be deposited.

An FDM part is akin to a laminate composite, with layers stacked vertically, in its structure and does exhibit similar behaviour in terms of directional properties. The thermo-mechanical properties of the components are not only dependent on varied characteristics of the material used in the filament but also the direction of the build. [3]

\subsection{Acrylonitrile Butadiene Styrene (ABS)}

FDM is used to fabricate Acrylonitrile Butadiene Styrene (ABS) parts of the highest quality. ABS is a common polymer that allows the user to execute functional tests on trial parts. ABS is an engineering plastic that has butadiene, a monomer for synthetic rubber, distributed uniformly over a matrix of acrylonitrile-styrene. [4]

It retains excellent toughness, easy processing ability, chemical resistance, good dimensional stability and is inexpensive. However, it suffers from intrinsic limitations in terms of mechanical strength.

Furthermore, it is easily fretted and non-conducting. Fretting as per the ASM is the wear experienced by two materials which are in contact at an inter-laminar level and subject to loads \& vibrations. Apart from ABS, polycarbonate, PC-ABS thermoplastic, ABS plus thermoplastic, PC-ISO thermoplastic, ABS-M30 thermoplastic, ULTEM 9085, PPSF/PPSU thermoplastic and ABS M30i thermoplastic are also used. [5]

\subsection{Rapid Tooling}

Rapid Tooling (RT) is a subset of AM, which has a significant impact on the product design \& manufacturing process mostly in the plastic processing industry. The cost effective \& time efficient nature of AM has proved to be a boon to the tooling industry as it has helped ensure the transfer of technology in the polymer industry. The process of creating tooling straight from CAD models, without intermediate steps is regarded as a significant step in reducing cost \& time to market in new product development. It also indicates a substantial shift in the tooling industry. [1]

Vacuum, sand \& investment casting along with injection moulding are now commercially using Rapid Casting (RC) through FDM as a means of creating tooling. RP is also now an aid to engineering design, planning, analysis, tooling and manufacturing through the printing of silicone rubber patterns and moulds. [1]

\subsection{Characteristics of Finished Parts}

The advantage of FDM is the increased freedom of design given to the engineers, as the movement of the extrusion head is controlled using ISO codes similar to CNC codes which have been used for decades. This increased accessibility pushes the capabilities FDM to many new fields of technology [6]

The principle of the FDM process involving three steps, namely, Preprocessing, Production \& Post processing.

- Preprocessing: This involves the use of a software tool to slice the 3D .stl (Standard Tessellation Language) file into layers of uniform thickness.

- Production: The extrusion head moves through the build volume and deposits the polymer. If supports are required, the support nozzle, which is part of the extrusion head, deposits an easily breakable or water soluble material on to the build platform.

- Post processing: The operator either removes the support or dissolves it in water with or without a catalyst. [7] 
- $\quad$ FDM can print components with plastics that have properties similar to a moulded component. Control over these properties is particularly is essential to create products that can be used as prototypes for functional tests. [8]

- $\quad$ Strength - Parts made with ABS have strengths that are $85 \%$ of moulded part made with ABS.

- Restricted accuracy - Filament shape (diameter of $1.27 \mathrm{~mm}$ ) of the material deposition inherently reduces the accuracy of the component built. Some of the newer machines have attempted to overcome these issues through an increased control of the machine parameters.

- Ease of material change - The material used to create the model is supplied in the form of a spool of filament. The machines can also keep track of the amount of material used in every process. These two factors make very easy to have adequate material for printing and reduces the time for material change drastically.

- Surface Roughness - The biggest disadvantage of FDM is the poor surface finish of the printed parts. Roughness is influenced by different parameters viz. part build orientation, layer thickness and raster angle. [9]

Some research has shown that the reduction in layer thickness increases the tensile strength, however, it is more expensive as more material is needed for manufacturing the components.

The orientation of the part being built plays a significant role as noted from the results of a Canadian research group. It was observed that maximum tensile strength is obtained for part orientation laid out to coincide with the direction of tensile loading. [10]

Essentially, many reviews have shown that there are numerous variables that affect the mechanical strength and surface characteristics of the FDM parts and further study is needed to provide greater clarity in the matter. [11]

\section{TESTING OF PRINTED PARTS}

\section{1: Sample Preparation}

The first step in creating a procedure to improve the surface characteristics \& properties of a $3 \mathrm{D}$ printed ABS part is to prepare samples for testing. It starts with a 3D model which is converted into STL (Standard Triangulation Language) file format in the CAD software. This format represents a 3D surface as an assembly of planar triangles. The file is imported into a pre-processing software. The pre-processing software calculates sections and slices the part design into number of horizontal layers, determines the amount of build and support material required for the part and orientation of the part on worktable. On the basis of part geometry and position, the support structure is created as needed.

The tool path or part printing instructions are generated by software. The FDM nozzle head follows the instructions from the software while printing the parts. All the samples are printed in accordance with the tests that need to be performed.

Shore D Hardness test for plastics requires the samples to be printed as per ASTM D2240 (length of $25.4 \mathrm{~mm}$, width of $12.7 \mathrm{~mm} \&$ thickness of $6.4 \mathrm{~mm}$ )

Izod Impact test for plastics requires the samples to be printed as per ASTM D256 (length of 63mm, width of 12.7 $\mathrm{mm}$, thickness of $3 \mathrm{~mm} \&$ depth after the notch of $10.16 \mathrm{~mm}$ ). The notch is created using a notch cutter after printing. Samples for the evaluation of surface roughness can be printed using either of the above standards.

All ABS specimens were printed using Statasys uPrint $\mathrm{SE}_{\text {Plus }}$. All samples were printed along X-direction. Statasys 
uPrint $\mathrm{SE}_{\text {Plus }}$ is set to print the samples at a temperature of $300{ }^{\circ} \mathrm{C}, 0.254 \mathrm{~mm}$ layer thickness and $45^{0} /-45^{0}$ raster angle. In order to vary parameters during pre-processing, an open source software is used. The temperature is changed to $280^{\circ} \mathrm{C}$ and $290^{\circ} \mathrm{C}$ and layer thickness is increased to $0.330 \mathrm{~mm}$ with the help of the software. The samples were printed along required orientation in different sets for various tests.

\section{2: Coating of FDM Parts}

In order to change the surface properties, the functional coating may be applied on the substrate of the material. The coating material is either used to improve the inherent property of the substrate or provide a characteristic that the substrate never had.

In this study, a chromium based paint is used to spray on to the substrate of ABS parts because:

- It is tough \& can be applied on a non-conductive material like ABS without any preparatory treatment.

- It is versatile because it can be coated on surfaces like wood \& glass.

- It is eco-friendly as it contains fewer toxins and is often water based

\section{Coating Procedure}

- Step 1: The painting process starts with removing dirt with a clean cotton cloth by wiping the surface.

- Step 2: Once the area is dry and clean, a polyurethane primer is applied on the surface with the help of a spray gun without making the layer too thick and allowed it to dry for some time.

- Step 3: After the part has dried, a putty (a powdered material applied to smoothen the base) is applied on the layer of primer. It is then allowed to dry for one hour.

- Step 4: Next step is to spray another layer of primer, before which sanding is done slowly to further avoid the peaks and valleys obtained after the putty is dried. Another layer of primer is sprayed to get a very bright surface finish and allow it to dry for another half an hour. All the above steps are done in room temperature and there is no requirement to change the temperature.

- Step 5: The part is then sprayed Krylon Premium Chrome paint. Spraying is done with the help of spray gun which is held 10 inches away from the surface of the part being sprayed. Once the coating is complete, the painted part is kept for 24 hours in an oven maintaining a temperature of $50{ }^{\circ} \mathrm{C}$.

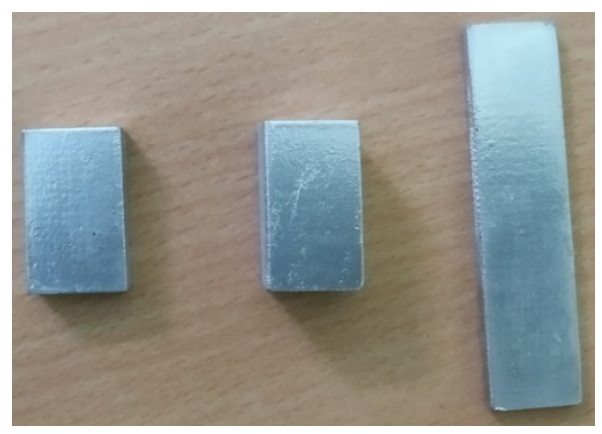

Figure 1: Uniform Chromium Coating on FDM 3D Printed Part. 
The figure 1 shows FDM parts with a uniform coating of chromium required for testing and comparison. The thickness obtained is around $0.25 \mathrm{~mm}$. Different sets of samples with different parameters were coated to test for strength and to study the surface properties. Figure 2 shows the set of samples coated to test and compare it with uncoated samples.

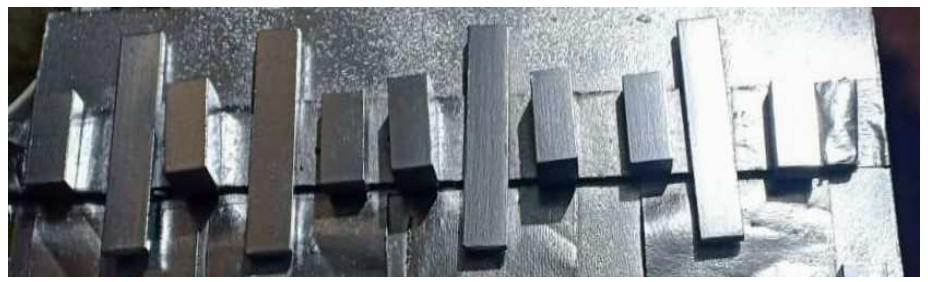

Figure 2: Different set of Samples Coated with Chromium Paint.

\section{3: Impact Tests}

The impact testing of ABS parts was conducted on Izod Impact testing machine by engaging a specimen mounted vertically. The material is subjected to a load from a weighted pendulum hammer striking the notch
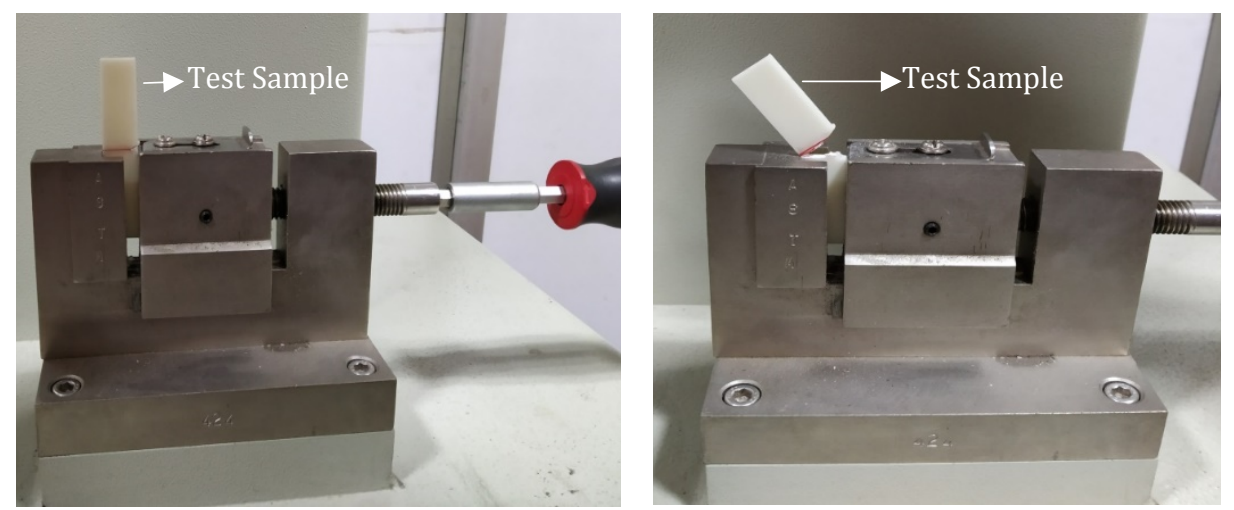

Figure 3: Breaking of Specimen after the Application of the Impact Load.

Impact energy was determined and the results are conveyed in terms of energy absorbed per unit of thickness at the notch or Impact strength of unit $\mathrm{J} / \mathrm{m}$. Figure 3 shows the broken specimen after the application of load from the weighted pendulum hammer.

Apparatus used: Testing Machine: International Equipment, Impact Energy of 0 to 25 joules, and Range of scales: 0 to $5.42 \mathrm{~J}, 150^{0}$ Release angle of pendulum, Hammer: R2 (Hard chrome plate).

The printed parts were tested for Impact Strength as per ASTM D256 under different conditions of temperature, layer thickness and coating. The results obtained are shown in tables $1 \& 2$

Table 1: Impact Test Results for Uncoated Samples

\begin{tabular}{|c|c|c|c|c|c|}
\hline SI No. & $\begin{array}{c}\text { Temp. } \\
\left({ }^{\mathbf{0}} \mathbf{C}\right)\end{array}$ & Layer Thickness(mm) & $\begin{array}{c}\text { Impact Energy } \\
(\mathbf{J})\end{array}$ & Angle & $\begin{array}{c}\text { Impact Strength } \\
(\mathbf{J} / \mathbf{m})\end{array}$ \\
\hline 1 & 300 & 0.254 & 0.35 & 138 & 116.6 \\
\hline 2 & 300 & 0.330 & 0.32 & 139 & 106.6 \\
\hline 3 & 290 & 0.330 & 0.3 & 142 & 100 \\
\hline 4 & 280 & 0.330 & 0.22 & 143 & 73.3 \\
\hline
\end{tabular}


Table 2: Impact Test Results for Coated Samples

\begin{tabular}{|c|c|c|c|c|c|}
\hline SI No. & $\begin{array}{c}\text { Temp. } \\
(\mathbf{0} \mathbf{c})\end{array}$ & Layer thickness(mm) & $\begin{array}{c}\text { Impact Energy } \\
(\mathbf{J})\end{array}$ & Angle & $\begin{array}{c}\text { Impact Strength } \\
(\mathbf{J} / \mathbf{m})\end{array}$ \\
\hline 1 & 300 & 0.254 & 0.4 & 137 & 133.3 \\
\hline 2 & 300 & 0.330 & 0.4 & 137 & 133.3 \\
\hline 3 & 290 & 0.330 & 0.22 & 143 & 73.3 \\
\hline 4 & 280 & 0.330 & 0.20 & 145 & 66.6 \\
\hline
\end{tabular}

The bar chart in figure 4 provides a comparison in terms of impact strength for different test samples. The highest Impact strength $(13.3 \mathrm{~J} / \mathrm{m})$ is obtained in components printed at $300^{\circ} \mathrm{C}$, with $0.254 \mathrm{~mm}$ layer thickness and with a coating of chromium.

\section{4: Hardness Tests}

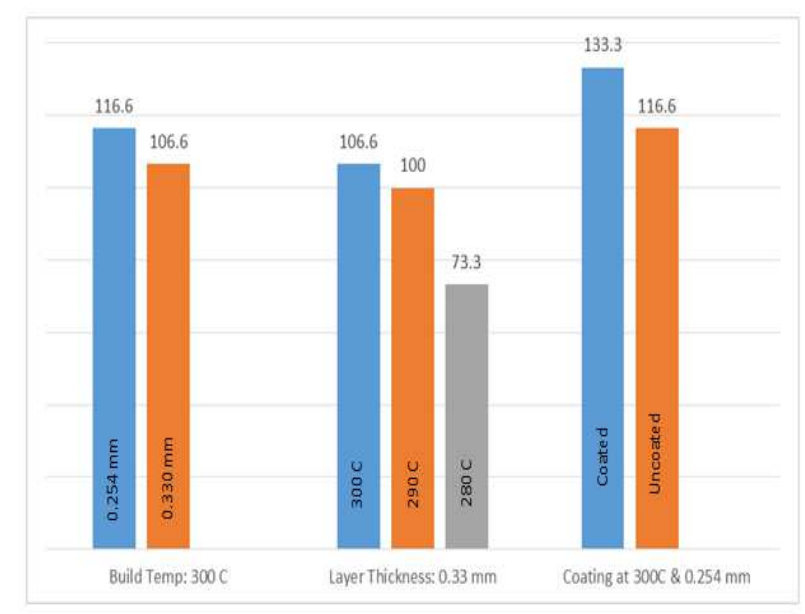

Figure 4: Chart Showing Values of Hardness Under Various Conditions of Temperature, Layer Thickness and Coating.

The most common measuring test for hardness of plastic is Shore (Durometer) test. The preferred hardness test method is the Shore Hardness which uses either Shore A or Shore D scale for elastometers or rubbers and softer plastics like vinyls, fluropolymers and polyolefins. Shore D \& A scales are used for harder \& softer plastics respectively. ${ }^{[12]}$

Figure 5 shows the instrument measuring the hardness of the coated specimen. Apparatus: Testing Machine: Durometer, Spring Power: $4450 \mathrm{cN}$, Indenter: Truncated cone $30^{\mathrm{O}}$ Measuring Distance: $3 \mathrm{~mm}$, Measuring Range: 0 to 100. The printed parts were tested for hardness as per ASTM D2240 under different conditions of temperature, layer thickness and coating.

The results obtained are shown in tables 3 and 4 .

Table 3: Hardness Test Values for Uncoated Samples

\begin{tabular}{|c|c|c|c|c|c|c|c|c|}
\hline \multirow{2}{*}{ Sl No. } & \multirow{2}{*}{$\begin{array}{c}\text { Temp. } \\
\left({ }^{0} \mathrm{c}\right)\end{array}$} & \multirow{2}{*}{ Layer Thickness(mm) } & \multicolumn{5}{|c|}{ Trials } & \multirow{2}{*}{ Average } \\
\hline & & & 1 & 2 & 3 & 4 & 5 & \\
\hline 1 & 300 & 0.254 & 69 & 70 & 70 & 70 & 70 & 69.6 \\
\hline 2 & 300 & 0.330 & 66 & 65 & 63 & 68 & 67 & 65.8 \\
\hline 3 & 290 & 0.330 & 65 & 62 & 61 & 62 & 64 & 62.8 \\
\hline 4 & 280 & 0.330 & 58 & 63 & 59 & 61 & 57 & 59.6 \\
\hline
\end{tabular}


Table 4: Hardness Test Values for Coated Samples

\begin{tabular}{|c|c|c|c|c|c|c|c|c|}
\hline \multirow{2}{*}{ SI No. } & Temp. & \multirow{2}{*}{ Layer Thickness(mm) } & \multicolumn{5}{|c|}{ Trials } & \multirow{2}{*}{ Average } \\
\cline { 4 - 8 } & & & $\mathbf{1}$ & $\mathbf{2}$ & $\mathbf{3}$ & $\mathbf{4}$ & $\mathbf{5}$ & \\
\hline 1 & 300 & 0.254 & 73 & 71 & 69 & 71 & 72 & 71.2 \\
\hline 2 & 300 & 0.330 & 70 & 68 & 71 & 68 & 70 & 69.4 \\
\hline 3 & 290 & 0.330 & 67 & 69 & 68 & 66 & 69 & 67.8 \\
\hline 4 & 280 & 0.330 & 59 & 64 & 65 & 64 & 65 & 63.4 \\
\hline
\end{tabular}

The chart in figure 6 shows that the highest hardness (71.2) is obtained in components printed at $300^{\circ} \mathrm{C}$, with $0.254 \mathrm{~mm}$ layer thickness and with a coating of chromium.

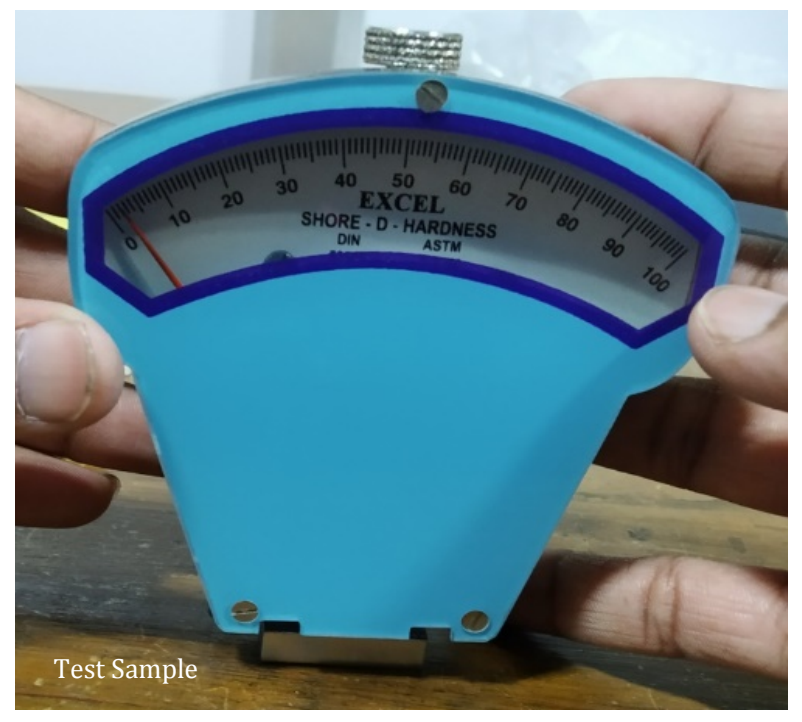

Figure 5: Measuring thr Hardness of the Coated Specimen.

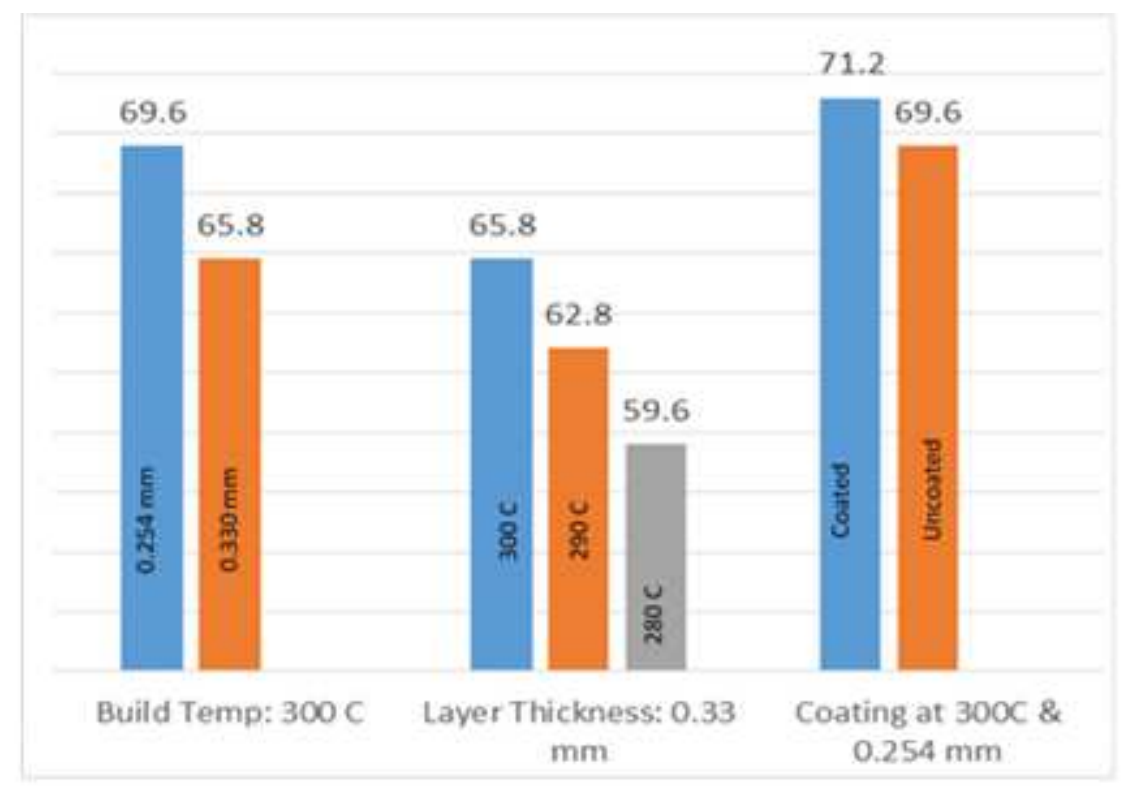

Figure 6: Chart Showing Values of Impact Strength under Various Conditions of Temperature, Layer thiCkness and Coating. 


\section{5: Measurement of Roughness}

The roughness is measured with the instrument called Metrix Microsurf Surface roughness tester. It is a portable device which measures the surface roughness very quickly, and gives the values in terms of average and depth. It consists of a probe which contacts the surface of the part and communicates with a computer as shown in the figure 7.

Apparatus: Testing Machine: Mertix Microsurf (Portable surface roughness tester), Stylus tip: Diamond 900/5 $\mu \mathrm{mR}$, Measuring range: $0.25,0.5,0.75 \mathrm{~mm} / \mathrm{s}, 1 \mathrm{~mm} / \mathrm{s}$ (returning), Power supply: 230 volts single phase $50 \mathrm{~Hz} \mathrm{AC}$, Evaluation parameters: $\mathrm{R}_{\mathrm{a}}, \mathrm{R}_{\mathrm{c}}, \mathrm{R}_{\mathrm{y}}, \mathrm{R}_{\mathrm{z}}, \mathrm{R}_{\mathrm{t}}, \mathrm{R}_{\max }, \mathrm{R}_{\mathrm{p}}, \mathrm{R}_{\mathrm{v}}, \mathrm{RSM}$

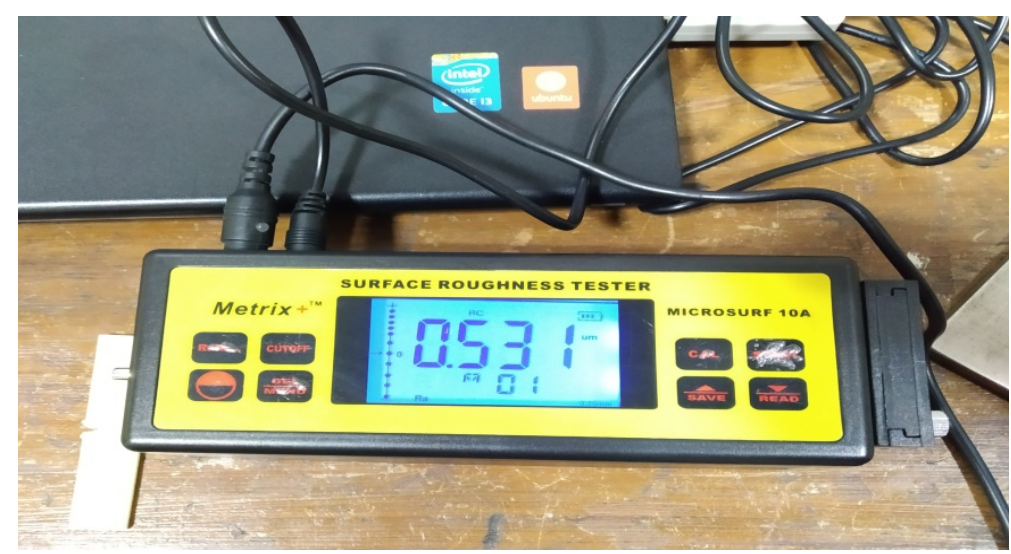

Figure 7: Measuring the Roughness of the Uncoated Specimen.

The results obtained are shown in tables 5 and 6

Table 5: Roughness Values for Uncoated Samples

\begin{tabular}{|c|c|c|c|c|c|c|}
\hline SI No. & $\begin{array}{c}\text { Temp. } \\
\left({ }^{\mathbf{0}} \mathbf{c}\right)\end{array}$ & Layer Thickness(mm) & $\begin{array}{c}\mathbf{R}_{\mathbf{a}} \\
(\boldsymbol{\mu \mathbf { m } )})\end{array}$ & $\begin{array}{c}\mathbf{R}_{\mathbf{z}} \\
(\boldsymbol{\mu \mathbf { m } )})\end{array}$ & $\begin{array}{c}\mathbf{R}_{\mathbf{q}} \\
(\boldsymbol{\mu} \mathbf{m})\end{array}$ & $\begin{array}{c}\mathbf{R}_{\mathbf{t}} \\
(\boldsymbol{\mu} \mathbf{m})\end{array}$ \\
\hline 1 & 300 & 0.254 & 0.491 & 1.39 & 0.489 & 1.404 \\
\hline 2 & 300 & 0.330 & 0.506 & 1.432 & 0.503 & 1.446 \\
\hline 3 & 290 & 0.330 & 0.518 & 1.467 & 0.513 & 1.481 \\
\hline 4 & 280 & 0.330 & 0.531 & 1.501 & 0.526 & 1.516 \\
\hline
\end{tabular}

Table 6: Roughness Values for Coated Samples

\begin{tabular}{|c|c|c|c|c|c|c|}
\hline SI No. & $\begin{array}{c}\text { Temp. } \\
\left({ }^{\mathbf{0}} \mathbf{c}\right)\end{array}$ & Layer Thickness $(\mathbf{m m})$ & $\begin{array}{c}\mathbf{R}_{\mathbf{a}} \\
(\boldsymbol{\mu m})\end{array}$ & $\begin{array}{c}\mathbf{R}_{\mathbf{z}} \\
(\boldsymbol{\mu m})\end{array}$ & $\begin{array}{c}\mathbf{R}_{\mathbf{q}} \\
(\boldsymbol{\mu m})\end{array}$ & $\begin{array}{c}\mathbf{R}_{\mathbf{t}} \\
(\boldsymbol{\mu m})\end{array}$ \\
\hline 1 & 300 & 0.254 & 0.247 & 0.7 & 0.245 & 0.706 \\
\hline 2 & 300 & 0.330 & 0.258 & 0.731 & 0.255 & 0.739 \\
\hline 3 & 290 & 0.330 & 0.345 & 0.975 & 0.342 & 0.985 \\
\hline 4 & 280 & 0.330 & 0.375 & 1.061 & 0.373 & 1.072 \\
\hline
\end{tabular}

The lowest value for surface roughness, $0.247 \mu \mathrm{m}$, is obtained in components printed at $300^{0} \mathrm{C}$, with $0.254 \mathrm{~mm}$ layer thickness and with a coating of chromium. The bar chart in figure 8 provides a comparison between the roughness values at different condition. 


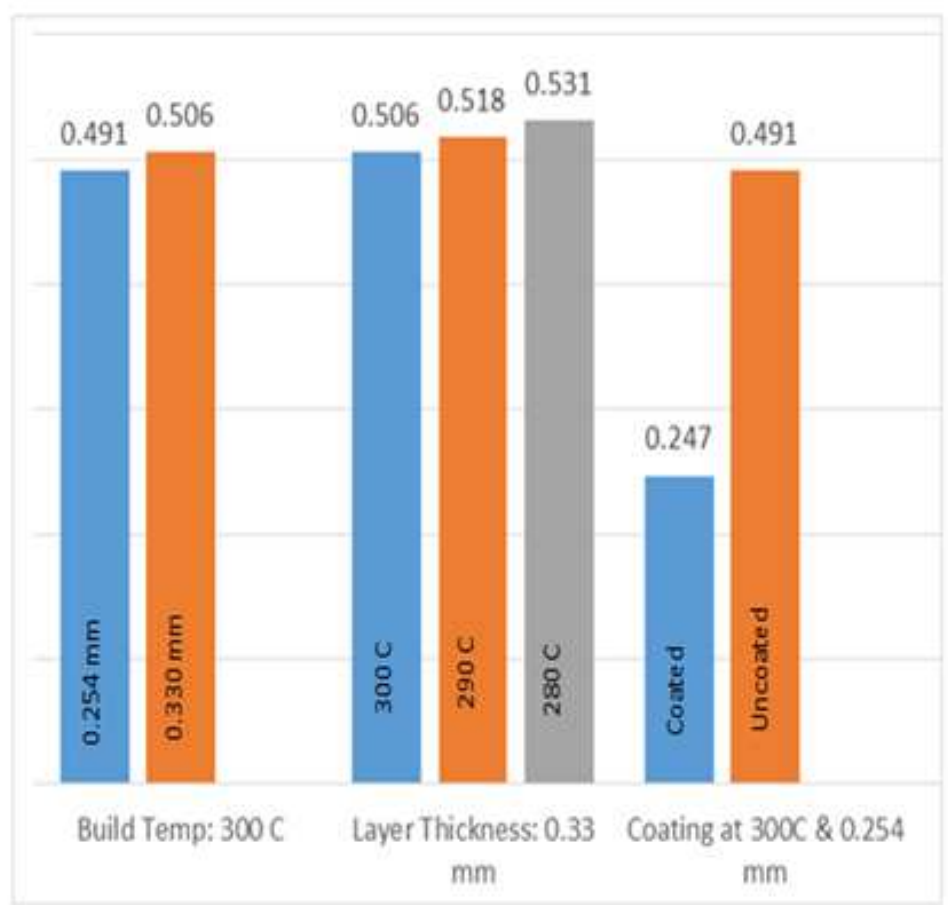

Figure 8: Chart Showing Values of Roughness under Various Conditions of Temperature,

\section{CONCLUSIONS}

Layer Thickness and Coating.

This study provides an insight into the impact of build parameters on the mechanical properties \& surface characteristics of ABS parts printed using FDM additive manufacturing technology. The optimum blend of parameters that exhibit an improvement in surface quality have been determined.

The tests for hardness \& impact strength and measurement of surface roughness have revealed that build temperature, layer thickness and secondary surface treatment has influenced the performance of the printed ABS parts. Build temperature directly correlates to the impact strength of the part. The hardness value decreases as the temperature decreases irrespective of the layer thickness but increases with the introduction of surface coating. When the samples are coated, the $\mathrm{R}_{\mathrm{a}}$ reduces to $50 \%$ compared to the uncoated parts. The surface roughness is better if the layer thickness 0.254 mm compared to that of $0.330 \mathrm{~mm}$.

It is evident that at a build temperature of $300^{\circ} \mathrm{C}$, with a layer thickness of $0.254 \mathrm{~mm}$ will provide the best possible output from pre-processing conditions. Across the board, the components have performed better when they have been coated with the chromium based paint indicating that it may be essential for rapid tooling applications

\section{REFERENCES}

1. Kamaljit Singh Boparai, Rupinder Singh \& Harwinder Singh; Development of rapid tooling using fused deposition modeling: A review; Rapid Prototyping Journal, March 2016, DOI: 10.1108/RPJ-04-2014-0048

2. S Dhaneshmand, C Aghanjafi \& A Ahmadi Nadooshan; The effect of chromium coating on RP technology for air foil 
manufacturing; Sadhana, Vol 35, Part 5, October 2010, pp. 569-584, Indian academy of Sciences

3. Constance Siemian, Mala Sharma and Sophia Ziemian; Anisotropic Mechanical Properties of ABS Parts Fabricated by Fused Deposition Modelling; 2012; DOI: 10.5772/34233

4. N. Sa'ude, M. Ibrahim, W. Saidin; Effect of Powder Loading and Binder Materials on Mechanical Properties in Iron-ABS Injection Molding Process; Applied Mechanics and Materials, Vol. 315, pp. 582-586, 2013

5. Ludmila Novakova-Marcincinova, Ivan Kuric "Basic and Advanced Materials for Fused Deposition Modeling Rapid Prototyping Technology” Manuf. and Ind. Eng., 11(1), 2012, ISSN 1338-6549.

6. Imtiyaz Khan, Dr. A. A. Shaikh “A Review of FDM Based Parts to Act as Rapid Tooling” International Journal of Modern Engineering Research ISSN: 2249-6645, Vol. 4, Iss.11, pp 59-64, Nov-2014

7. Adhiyamaan Arivazhagan, S.H. Masood "Dynamic Mechanical Properties of ABS Material Processed by Fused Deposition Modeling” International Journal of Engineering Research and Applications, Vol. 2, Issue 3, May-Jun 2012, pp.2009-2014

8. Ravi Patel, Satyam Patel, Jaimin Patel “A Review on Optimization of Process Parameter of Fused Deposition Modeling for Better Dimensional Accuracy" International Journal of Engineering Development and Research 2014 IJEDR, Volume 2, Issue 2, ISSN: 2321-9939.

9. Tejendrasinh S. Raol, Dr. K. G. Dave, Dharmesh B. Patel, Viral N. Talati “An Experimental Investigation of Effect of Process Parameters on Surface Roughness of Fused Deposition Modeling Built Parts International Journal of Engineering Research \& Technology, ISSN: 2278-0181, Vol. 3 Issue 4, April-2014

10. Godfrey C. Onwubolu and Farzad Rayegani, Characterization and Optimization of Mechanical Properties of ABS Parts Manufactured by the Fused Deposition Modelling Process; International Journal of Manufacturing Engineering, vol. 2014, Article ID 598531, 13 pages, 2014. doi:10.1155/2014/598531

11. Jasgurpreet Singh Chohan, Rupinder Singh,Pre and post processing techniques to improve surface characteristics of FDM parts: a state of art review and future applications, Volume 23, Number $3 \bullet 2017 \bullet 495-513$

12. Rupinder Singh, Alok Trivedi and Sunpreet Singh, Experimental investigation on shore hardness of barrel-finished FDM patterns, 19 January 2017, DOI 10.1007/s12046-017-0709-6 